Article

\title{
Investigating the Association between Wood and Charcoal Domestic Cooking, Respiratory Symptoms and Acute Respiratory Infections among Children Aged Under 5 Years in Uganda: A Cross-Sectional Analysis of the 2016 Demographic and Health Survey
}

\author{
Katherine E. Woolley ${ }^{1,+}{ }^{+}$, Tusubira Bagambe ${ }^{1,+}$, Ajit Singh $^{2}{ }^{\infty}$, William R. Avis ${ }^{3}$, \\ Telesphore Kabera ${ }^{4}{ }^{(0}$, Abel Weldetinsae ${ }^{5}{ }^{\circ}$, Shelton T. Mariga ${ }^{6}$, Bruce Kirenga ${ }^{6}$, \\ Francis D. Pope ${ }^{2} \mathbb{D}$, G. Neil Thomas ${ }^{1}$ and Suzanne E. Bartington ${ }^{1, * \mathbb{D}}$ \\ 1 Institute of Applied Health Research, University of Birmingham, Edgbaston, Birmingham B15 2TT, UK; \\ KEW863@student.bham.ac.uk (K.E.W.); TVB850@alumni.bham.ac.uk (T.B.); \\ G.N.Thomas@bham.ac.uk (G.N.T.) \\ 2 School of Geography, Earth and Environmental Sciences, University of Birmingham, Edgbaston, \\ Birmingham B15 2TT, UK; A.Singh.2@bham.ac.uk (A.S.); F.Pope@bham.ac.uk (F.D.P.) \\ 3 International Development Department, University of Birmingham, Edgbaston, Birmingham B15 2TT, UK; \\ W.R.Avis@bham.ac.uk \\ 4 College of Science and Technology, University of Rwanda, Avenue de l'Armee P.O. Box 3900, Rwanda; \\ kaberacris@yahoo.fr \\ 5 Ethiopian Public Health Institute, Addis Ababa P.O. Box 1242, Ethiopia; abelweldetinsae@gmail.com \\ 6 Makerere University Lung Institute, College of Health Sciences, Mulago Hospital, Kampala P.O. Box 7749, \\ Uganda; sheltontmariga@gmail.com (S.T.M.); brucekirenga@yahoo.co.uk (B.K.) \\ * Correspondence: s.bartington@bham.ac.uk; Tel.: +44-121-414-7742 \\ + Joint first author.
}

Received: 6 May 2020; Accepted: 27 May 2020; Published: 4 June 2020

Abstract: Background: Household air pollution associated with biomass (wood, dung, charcoal, and crop residue) burning for cooking is estimated to contribute to approximately 4 million deaths each year worldwide, with the greatest burden seen in low and middle-income countries. We investigated the relationship between solid fuel type and respiratory symptoms in Uganda, where $96 \%$ of households use biomass as the primary domestic fuel. Materials and Methods: Cross-sectional study of 15,405 pre-school aged children living in charcoal or wood-burning households in Uganda, using data from the 2016 Demographic and Health Survey. Multivariable logistic regression analysis was used to identify the associations between occurrence of a cough, shortness of breath, fever, acute respiratory infection (ARI) and severe ARI with cooking fuel type (wood, charcoal); with additional sub-analyses by contextual status (urban, rural). Results: After adjustment for household and individual level confounding factors, wood fuel use was associated with increased risk of shortness of breath (AOR: 1.33 [1.10-1.60]), fever (AOR: 1.26 [1.08-1.48]), cough (AOR: 1.15 [1.00-1.33]), ARI (AOR: 1.36 [1.11-1.66] and severe ARI (AOR: 1.41 [1.09-1.85]), compared to charcoal fuel. In urban areas, Shortness of breath (AOR: 1.84 [1.20-2.83]), ARI (AOR: 1.77 [1.10-2.79]) and in rural areas ARI (AOR: 1.23 [1.03-1.47]) and risk of fever (AOR: 1.23 [1.03-1.47]) were associated with wood fuel usage. Conclusions: Risk of respiratory symptoms was higher among children living in wood compared to charcoal fuel-burning households, with policy implications for mitigation of associated harmful health impacts.

Keywords: acute respiratory infection; biomass fuel; household air pollution; respiratory symptoms; Uganda 


\section{Introduction}

Globally, almost 3 billion people utilize biomass fuels such as wood, charcoal, animal dung, and plant residues for everyday domestic activities including cooking and heating [1,2]. These fuels are typically burned in simple stoves or open fires, with inefficient combustion associated with the release of hazardous gases and particles such as carbon monoxide $(\mathrm{CO})$, nitrogen dioxide $\left(\mathrm{NO}_{2}\right)$, and particulate matter (PM) [1,3]. The arising pollutants are the major contributors to Household Air Pollution (HAP), responsible for almost 4 million deaths worldwide each year, with the greatest burden in low and middle-income country (LMIC) settings, including sub-Saharan Africa [2]. Chronic HAP exposure associated with biomass fuel combustion is recognized to be detrimental to human health, with consistent evidence for an association with respiratory and cardiovascular disease conditions including acute respiratory tract infections (ARIs) [4], asthma [5], chronic obstructive pulmonary disease (COPD) [6], lung cancer [7], tuberculosis [6], stroke, ischemic heart disease [2] and metabolic and cognitive health [8]. Moreover, families living in rural households and those engaged in subsistence agriculture are recognized to experience a higher HAP morbidity and mortality burden, compared to those living in urban LMIC settings [9-11].

Uganda is a landlocked country in East Africa, with an increasing population of 43 million and a gross domestic product (GDP) per capita of approximately $\$ 643$ USD in 2018, 24.2\% of which is generated by the agricultural sector [12]. Uganda has a population density of 231 people per $\mathrm{km}^{2}$ with an urban population of 10 million $(23.3 \%)$ in 2018, with growth of $6.2 \%$ since 2017; a significant proportion of which live in or around the Ugandan capital Kampala. The life expectancy of Uganda is 62.5 years, with an under five-mortality rate of 46.4 children per 1,000 live births [13]; both comparable to other East African countries.

Prevailing reliance upon biomass energy in sub-Saharan Africa also presents broader implications for achieving sustainable development, including fiscal costs imposed by morbidity and mortality, opportunity costs of fuel collection and environmental degradation through deforestation and carbon dioxide emissions [14]. In Uganda, East Africa, it is estimated that $92 \%$ of the total energy demand comes from biomass, with $96 \%$ of domestic cooking being undertaken on firewood and charcoal, resulting in annual wood consumption of 20 million tons [15]. HAP is a major priority risk factor for Uganda, responsible for $2.93 \%$ of deaths, including $1.47 \%$ of deaths due to respiratory disease [16]. Access to cleaner fuels, such as electricity and Liquid Petroleum Gas (LPG), is limited due to logistical and financial supply barriers particularly high capital infrastructure costs, most notably in rural areas; therefore contributing to prevailing health and social inequity [15].

Limited existing epidemiological evidence from Uganda suggests an association between use of biomass fuels for cooking and incidence of chronic respiratory and cardiovascular disease [17,18]; however few studies have investigated the relationship between solid fuel usage for cooking and incidence of respiratory symptoms in pre-school aged children. Most existing research has focused upon health impacts among those living in rural areas and to the best of our knowledge there is no previous study of respiratory symptoms associated with charcoal and wood fuel cooking among a nationally representative population sample, including both rural and urban biomass fuel households. The present study therefore aims to identify the association between risk of respiratory symptoms (cough, fever and short rapid breaths), ARI and severe ARI (Infection of the upper or lower respiratory tract presenting with cough, shortness of breath and fever) [19] with charcoal and wood fuel cooking among children aged under 5 years in Uganda, using data obtained from the population-based Demographic and Health Survey (DHS).

\section{Materials and Methods}

\subsection{Setting and Study Design}

Data for this cross-sectional study were obtained from the most recently available nationally representative Ugandan DHS [20], a population level survey implemented by the Uganda Bureau 
of Statistics (UBOS, supported by USAID, UNICEF, UNFPA) from 20 June to 28 December 2016 [21]. Two-stage stratified sampling was applied to identify eligible residential households across 697 enumeration areas (average 130 households) from 112 Districts and 15 regions in Uganda. From 20,791 eligible households, 18,506 resident ever-married women age 15-49 years were interviewed (98\% response rate) [21]. Institutional living arrangements (e.g., boarding schools, police camps, army barracks, and hospitals) were excluded; as were households with no response at the time of fieldwork completion.

Survey questionnaires were modified from those within the Phase VII DHS Program model, adapted to reflect the population and health issues relevant to Uganda. Information for this study was obtained from the (i) household questionnaire; comprising information on household structure, socio-demographic and housing characteristics, including domestic cooking fuel type, and; (ii) children's questionnaire; including questions on maternal and child health outcomes and lifestyle characteristics. All survey fieldwork was undertaken within the participant's home, by trained local fieldworkers supervised by senior staff, with data entry directly to tablet computers transferred to the UBOS central processing office by a secure internet system.

\subsection{Ethical Approval and Authorization}

Ethical approval for primary data collection was provided by the Uganda Ministry of Health. The investigators obtained the anonymised, aggregate data from the publicly available DHS online data archive [20] with authorization granted for data access for this current investigation on 16 June 2019.

\subsection{Data Variables}

\subsubsection{Measures of Respiratory Symptoms and Acute Respiratory Infections}

To assess respiratory symptoms, maternal respondents were asked if each of their children aged under 5 years had experienced the following symptoms within the two weeks prior to the survey: (i) a cough (ii) short rapid breaths or difficulty breathing (iii) a fever; each categorized and modelled as binary outcome measures (yes, no). A composite measure was created for both ARI and severe ARI, reflecting the presence of respiratory symptoms with or without fever, with each separately modelled as a binary health outcome measure (yes, no). ARI was classified as present of cough and short rapid breaths/difficulty [22], whereas severe ARI was composed of the presence of all three of these symptoms, (e.g., cough, short rapid breaths/difficulty breathing and fever) [23].

\subsubsection{Measure of Exposure to HAP}

Among those households in which cooking activities were performed, self-reported cooking fuel types were identified from the household dataset and categorized as "cleaner fuels" (electricity, LPG, natural gas, biogas); "Solid biomass fuels and kerosene" (kerosene, coal/lignite, charcoal, wood, straw/shrubs/grass, agricultural crop, animal dung) or other fuel types. Those mother-child pairs living in households reporting wood and charcoal fuel cooking were extracted for further analyses.

\subsubsection{Child and Maternal Characteristics}

Characteristics of household children comprised; age (0-11, 12-23, 24-35, 36-48, 48-59 months), sex (male, female), birthweight ( $\mathrm{kg}$, by maternal recall), weight for height (z score), mode of delivery (caesarean, vaginal), birth order (first, not first born), breastfeeding status (ever, never), vitamin A supplementation in the last 6 months (yes, no), iron supplementation (yes, no). Those children with diagnosed mild, moderate or severe anaemia $(n=2139)$ were excluded from further analyses, due to anaemia being a known factor for increased ARI risk [24], which could not be accounted for in the adjusted analyses due to the high quantity of missing data (2139/15405; 13.9\%). Maternal characteristics included age (15-24, 25-35, 36-49 years) and highest attained educational level (none, primary, secondary/higher). 


\subsubsection{Household and Geographical Characteristics}

Household characteristics were accounted for by the following variables: number of household members, indoor household smoking (yes, no), cooking location (inside, outdoors). Season at the time of DHS contact was determined from the month of interview and classified as dry (June to August) or wet (September to November) using information from the Central Intelligence Agency (CIA) fact book [25]. The five category DHS wealth index was used as a measure of household level socio-economic status (lowest, low, middle, high, highest). This composite measure reflects household ownership of selected assets (e.g., television, bicycle, car), dwelling characteristics (e.g., source of drinking water, sanitation facilities, types of cooking fuel, and floor material), with assessment of relative wealth category calculated by principle components analysis.

Contextual characteristics comprised: place of residence (rural, urban), and country region (Kampala, South Buganda, North Buganda, Busoga, Bukedi, Bugisu, Teso, Karamoja, Lango, Acholi, West Nile, Bunyoro, Tooro, Ankole, Kigez). DHS classifies rural and urban area, as per the country of survey; in this case Uganda uses enumeration areas are defined as being rural or urban. Urban areas are defined as officially approved cities, municipalities, town councils and town boards [21], at the time which the survey was undertaken.

\subsection{Data Analysis}

All data processing, manipulation and analyses was performed using R studio [26]. Descriptive statistics were summarized by number of cases $(n)$, percentages (\%) (categorical variables) and median and interquartile range (IQR) (continuous variables). The association between fuel type (wood vs. charcoal) and respiratory health outcomes (cough, fever, short rapid breaths or ARI/severe ARI), was determined through multivariable logistic regression analysis; reporting the odds ratio (OR), 95\% Confidence interval ( $95 \% \mathrm{CI}$ ) and level of significance ( $p$-value). Univariable forward selection was used to determine variables for inclusion in the adjusted analysis. Covariates include, child's age, sex, birth order, mode of delivery, vitamin A supplementation, breastfeeding, iron supplementation, maternal age, maternal education, wealth index, household smoking, cooking location, number of household remembers, season, place of residence, region. Statistical significance in the adjusted model was set at $p<0.05$. Model collinearity was checked using variance inflation factors (VIF function in $\mathrm{R}$ ). The primary analysis was performed upon the whole dataset, with subsequent sub-analyses by rural and urban area status respectively.

\section{Results}

A total of 19,588 households were included in the 2016 DHS survey in Uganda, at a 98\% response rate. Data were collected for 15,522 children under five years. A total $0.3 \%$ of children resided in a households using 'cleaner' fuels (0.2\%-electricity, 0.1\%—LPG, $0.04 \%$-Biogas) and $99.3 \%$ using biomass as the main source of cooking fuel; the majority $(78.6 \%)$ using wood, charcoal $(21.4 \%)$. Of these children, we excluded those with diagnosed anaemia (13.9\%). Among charcoal and wood fuel households, $93 \%$ had complete information regarding the outcome variables: presence of cough and shortness of breath within the last two weeks (Table 1). Of this study population, the median child age was 30 months (IQR: $14-45$ ) with $81.7 \%$ residing in a rural location.

Within rural study areas, wood fuel use was used by a higher proportion of respondents $(87.5 \%)$ compared to urban areas (35.9\%), when compared to charcoal fuel. The rural study population had a median age of 30 months (IQR: 14-45 months) compared to 31 months (IQR: $15-45$ months) in the urban population. 
Table 1. Biomass fuel status (all study households) by respiratory outcome measures (frequency, percentage).

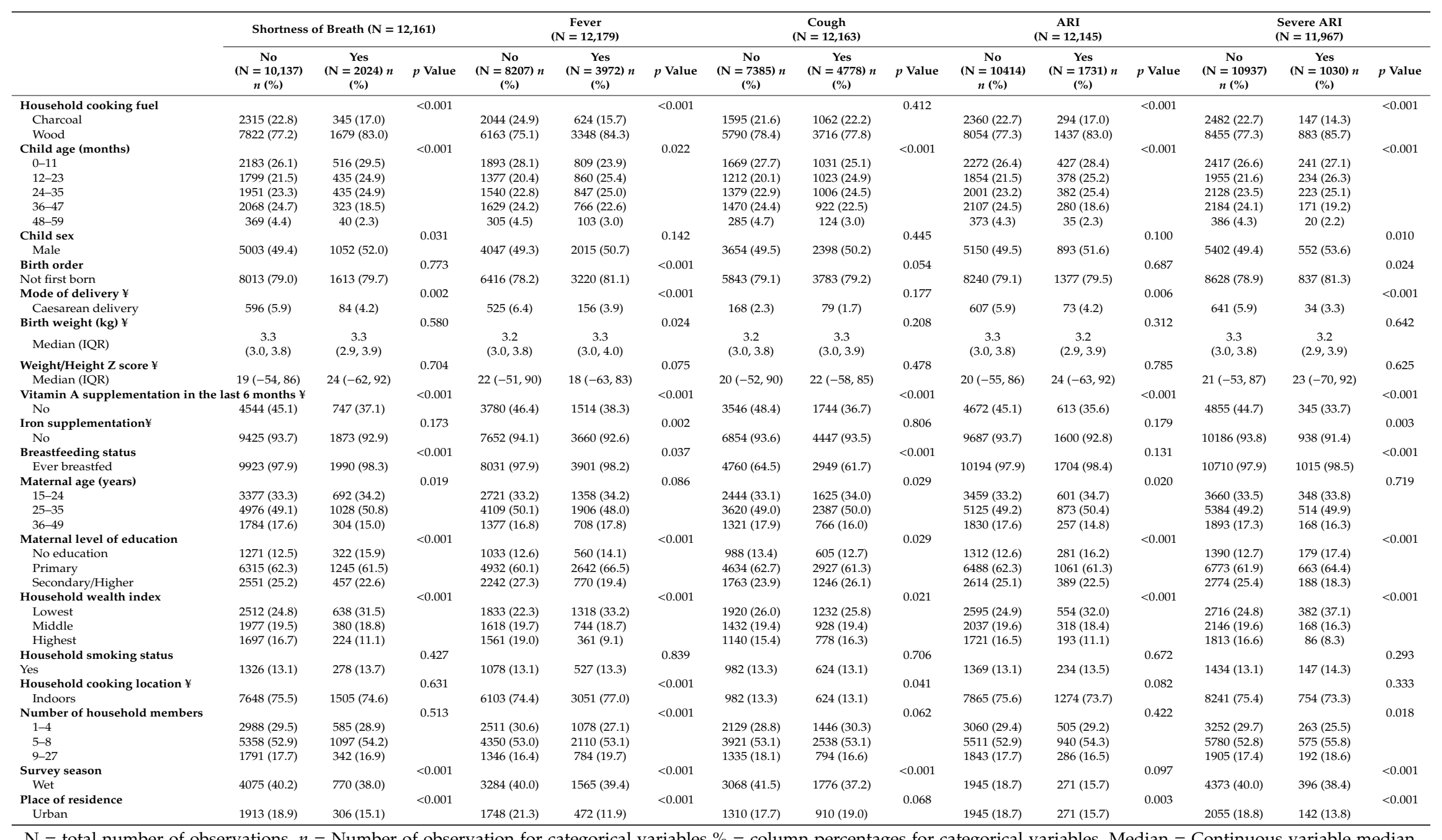

$\mathrm{N}=$ total number of observations, $n=$ Number of observation for categorical variables $\%=$ column percentages for categorical variables, Median $=$ Continuous variable median, $\mathrm{IQR}=$ Interquartile Range, $p$ value $=$ Chi-Squared for categorical variables, and Kruskal Wallis for Continuous variables. $¥$ contains missing observations; Received Vitamin $\mathrm{A}=84(0.7 \%)$; Birth weight $=3750(30.8 \%)$; Height/Weight $=9900(81.3 \%)$; Cooking location $=17(0.1 \%)$; Mode of delivery $=49(0.4 \%)$; Taking iron supplements $=90(0.7 \%$-Fever $)$. 


\subsection{Shortness of Breath in the Past Two Weeks}

Shortness of breath was reported among $17.7 \%$ of children living in wood fuel households compared to $13.0 \%$ in charcoal households $(p<0.001)$ (Table 2). After adjustment for confounding factors (wealth index, season, region, place of residence household size, maternal level of education, breastfeeding status, mode of delivery, vitamin A supplementation and iron supplementation) shortness of breath was significantly associated with cooking fuel type, with an observed increased risk associated with wood fuel (adjusted OR (AOR): 1.33; 95\% CI: 1.10-1.60; $p<0.01$ ) compared to charcoal cooking. Other factors associated with significantly increased risk in adjusted analyses were male sex (AOR: 1.13; 95\% CI: $1.02-1.26 ; p<0.05$ ) and vaginal delivery (AOR: 1.39; 95\% CI: 1.08-1.83; $p<0.05$ ).

\subsection{Cough in the Past Two Weeks}

Cough was reported among $40.0 \%$ of children residing in charcoal cooking households, compared to $39.1 \%$ in wood fuel households $(p=0.412)$. In the adjusted analysis, risk of cough was significantly higher among those living in wood (AOR: 1.15; 95\% CI: 1.00-1.33; $p<0.05$ ), compared to charcoal cooking fuel households. Other observed factors associated with increased risk of cough were vaginal delivery (AOR: 1.27; 95\%CI: 1.06-1.54; $p<0.05$ ), vitamin A supplementation (AOR: $1.67 ; 95 \%$ CI: 1.53-1.83; $p<0.001$ ), outdoor cooking (AOR: 1.13; 95\% CI: 1.02-1.25; $p<0.05$ ) and dry season (OR: 1.18; 95\% CI: $1.08-1.28 ; p<0.001)$.

\subsection{Fever in the Past Two Weeks}

Of those children who resided in wood fuel households, $35.2 \%$ were reported to exhibit a fever compared to $23.4 \%$ in charcoal cooking households $(p<0.001)$, which remained significant after adjustment for confounding factors (AOR: 1.26 ; 95\% CI: $1.08-1.48 ; p<0.01$ ). In adjusted analyses, risk of fever was also significantly associated with vitamin A supplementation (AOR: $1.48 ; 95 \% \mathrm{CI}$ : 1.34-1.63; $p<0.001$ ) and vaginal delivery (AOR: 1.38; 95\% CI: 1.11-1.73); $p<0.01$ ), with reduced risk among children of mothers with a higher level of education (AOR: $0.80 ; 95 \%$ CI: $0.71-0.91: p<0.001$ ) or highest wealth index (AOR: $0.64 ; 95 \%$ CI: $0.51-0.81 ; p<0.001$ ). Risk of fever was highest among those children aged 12-24 months, with a reduced risk among infants in the first year of life (AOR $0.67 ; 95 \%$ CI: $0.59-0.77: p<0.001$ ) and those between 24 months and 5 years of age.

3.4. ARI (Shortness of Breath and Cough in the Past Two Weeks) and Severe ARI (Shortness of Breath, Cough and Fever in the Past Two Weeks)

ARI was observed within $15.1 \%$ of children in wood fuel compared to $11.1 \%$ of charcoal fuel households. In the adjusted analysis wood fuel was observed to be associated with increased ARI risk (AOR: 1.36; 95\% CI: 1.11-1.66); $p<0.01$ ), as was vaginal delivery (AOR: $1.3795 \%$ CI: $1.04-1.83 ; p<0.05$ ) and vitamin A supplementation (AOR: 1.58; 95\% CI: 1.40-1.78; $p<0.001$ ).

Among wood fuel households, $9.5 \%$ of children were reported to have shown symptoms of severe ARI compared to $5.6 \%$ of those living in charcoal fuel households $(p<0.001)$. After adjustment for confounding factors, wood fuel was observed to be associated with increased severe ARI risk (AOR: $1.41 ; 95 \%$ CI: 1.09-1.85; $p<0.05$ ), as was male sex (AOR: 1.19; 95\% CI: $1.04-1.38 ; p<0.05$ ), vaginal delivery (AOR: $1.5195 \%$ CI: 1.04-2.28; $p<0.05$ ) and vitamin A supplementation (AOR: 1.67; 95\% CI: $1.43-1.96 ; p<0.001)$. Older children aged between 36 months and 5 years also had reduced ARI and severe ARI risk compared to those aged between 12 and 23 months.

\subsection{Urban and Rural Population}

Regression analyses were repeated separately for rural and urban areas. Among those living in urban areas, shortness of breath was reported among $18.9 \%$ of children in wood fuel households compared to $1.09 \%$ in charcoal fuel households $(p<0.001)$, with wood fuel being observed to increase the risk of shortness of breath (AOR: 1.84; 95\% CI: 1.20-2.83; $p<0.01$ ) and ARI (AOR: $1.77 ; 95 \%$ CI: 
$1.10-2.79 ; p<0.05)$ compared to charcoal fuel (Table 3). Conversely, no significant association was observed between cooking fuel type and risk of cough, fever or severe ARI, although absolute effect sizes were consistent with those for the combined analysis. In addition, within the rural population there was only an observed association between wood fuel type and risk of fever (AOR: $1.23 ; 95 \% \mathrm{CI}$ : 1.03-1.47; $p<0.05$ ) and ARI (AOR: 1.27; 95\% CI: 1.01-1.59; $p<0.05$ ) (Table 3). 
Table 2. Adjusted Logistic regression analysis for the whole study population $(\mathrm{N}=13,266)$.

\begin{tabular}{|c|c|c|c|c|c|c|c|c|c|c|c|c|c|c|c|}
\hline \multirow[b]{2}{*}{ Predictor $(\mathrm{N})$} & \multicolumn{3}{|c|}{$\begin{array}{l}\text { Shortness of Breath } \\
(N=12,161)\end{array}$} & \multicolumn{3}{|c|}{$\begin{array}{c}\text { Cough } \\
(\mathrm{N}=12,163)\end{array}$} & \multicolumn{3}{|c|}{$\begin{array}{c}\text { Fever } \\
(\mathrm{N}=12,179)\end{array}$} & \multicolumn{3}{|c|}{$\begin{array}{c}\text { ARI } \\
(\mathrm{N}=12,145)\end{array}$} & \multicolumn{3}{|c|}{$\begin{array}{l}\text { Severe ARI } \\
(N=11.967)\end{array}$} \\
\hline & $\%+$ & OR & CI & $\%+$ & OR & CI & $\%+$ & OR & CI & $\%+$ & OR & CI & $\%+$ & OR & CI \\
\hline \multicolumn{16}{|l|}{ Household cooking fuel } \\
\hline Charcoal (2660) & 13.0 & $\operatorname{Ref}$ & & 40.0 & Ref & & 23.4 & Ref & & 11.1 & & Ref & 5.6 & Ref & \\
\hline Wood (9501) & 17.7 & $1.33^{b}$ & $1.10-1.60$ & 39.1 & $1.15^{\mathrm{a}}$ & $1.00-1.33$ & 35.2 & $1.26^{\mathrm{b}}$ & $1.08-1.48$ & 15.1 & $1.36^{\mathrm{b}}$ & $1.11-1.66$ & 9.5 & $1.41^{\mathrm{a}}$ & $1.09-1.85$ \\
\hline \multicolumn{16}{|l|}{ Child age (months) (3972) } \\
\hline $0-11(2707)$ & 19.1 & 1.09 & $0.94-1.27$ & 38.2 & $0.83^{\mathrm{b}}$ & $0.73-0.93$ & 29.9 & $0.67^{\mathrm{c}}$ & $0.59-0.77$ & 15.8 & 1.04 & $0.89-1.22$ & 9.1 & 0.93 & $0.76-1.13$ \\
\hline $12-23(2237)$ & 19.5 & Ref & & 45.8 & Ref & & 38.4 & Ref & & 16.9 & & Ref & 10.7 & Ref & \\
\hline $24-35$ (2387) & 8.2 & 0.94 & $0.80-1.09$ & 42.4 & 0.89 & $0.79-1.01$ & 35.5 & $0.85^{\mathrm{a}}$ & $0.75-0.97$ & 16.0 & 0.95 & $0.81-1.12$ & 9.5 & 0.87 & $0.71-1.06$ \\
\hline $36-47$ (2395) & 13.5 & $0.64^{\mathrm{c}}$ & $0.54-0.76$ & 38.5 & $0.77^{\mathrm{c}}$ & $0.68-0.87$ & 32.0 & $0.72^{\mathrm{c}}$ & $0.63-0.83$ & 11.7 & $0.66^{c}$ & $0.55-0.78$ & 7.3 & $0.65^{\mathrm{c}}$ & $0.52-0.80$ \\
\hline $48-59(408)$ & 9.8 & $0.47^{\mathrm{c}}$ & $0.32-0.66$ & 30.3 & $0.55^{\mathrm{c}}$ & $0.43-0.69$ & 25.2 & $0.50^{\mathrm{c}}$ & $0.38-0.65$ & 8.6 & $0.51^{\mathrm{c}}$ & $0.35-0.73$ & 4.9 & $0.47^{b}$ & $0.28-0.74$ \\
\hline \multicolumn{16}{|l|}{ Child sex } \\
\hline Female (6106) & 15.9 & Ref & & 38.9 & & & 32.0 & Ref & & 14.8 & & Ref & 7.9 & Ref & \\
\hline Male (6055) & 17.4 & $1.13^{\mathrm{a}}$ & $1.02-1.26$ & 39.6 & 1.06 & $0.98-1.15$ & 33.2 & 1.07 & $0.98-1.18$ & 13.7 & 1.12 & $1.00-1.25$ & 9.3 & $1.19^{\mathrm{a}}$ & $1.04-1.38$ \\
\hline \multicolumn{16}{|l|}{ Mode of delivery $¥$} \\
\hline Caesarean section (680) & 12.4 & Ref & & 36.9 & & & 22.9 & Ref & & 14.5 & & Ref & 5.0 & Ref & \\
\hline Vaginal delivery $(11,298)$ & 16.9 & $1.39^{\mathrm{a}}$ & $1.08-1.83$ & 39.5 & $1.27^{\mathrm{a}}$ & $1.06-1.54$ & 33.2 & $1.38^{\mathrm{b}}$ & $1.11-1.73$ & 10.7 & $1.37^{\mathrm{a}}$ & $1.04-1.83$ & 8.8 & $1.51^{\mathrm{a}}$ & $1.04-2.28$ \\
\hline \multicolumn{16}{|c|}{ Vitamin A supplementation in last 6 months $¥$} \\
\hline No (5291) & 14.1 & Ref & & 33.0 & & & 28.6 & Ref & & 11.6 & & Ref & 6.6 & Ref & \\
\hline Yes (6792) & 8.7 & $1.47^{\mathrm{c}}$ & $1.31-1.64$ & 44.3 & $1.67^{\mathrm{c}}$ & $1.53-1.83$ & 35.8 & $1.48^{\mathrm{c}}$ & $1.34-1.63$ & 16.3 & $1.58^{\mathrm{c}}$ & $1.40-1.78$ & 10.1 & $1.67^{\mathrm{c}}$ & $1.43-1.96$ \\
\hline \multicolumn{16}{|l|}{ Breastfeeding status } \\
\hline Ever $(11,932)$ & 16.7 & Ref & & 39.4 & Ref & & 32.7 & Ref & & 14.3 & & Ref & 8.7 & Ref & \\
\hline Never (247) & 13.7 & 0.81 & $0.50-1.26$ & 32.0 & $0.71^{\mathrm{a}}$ & $0.51-0.98$ & 28.7 & 0.91 & $0.63-1.29$ & 10.9 & 0.76 & $0.45-1.22$ & 6.2 & 0.71 & $0.33-1.34$ \\
\hline \multicolumn{16}{|l|}{ Maternal age (years) (3972) } \\
\hline $15-24(4079)$ & 17.0 & $\operatorname{Ref}$ & & 39.9 & Ref & & 33.3 & Ref & & 14.8 & & Ref & 8.7 & $\operatorname{Ref}$ & \\
\hline $25-35$ (6015) & 17.1 & 1.07 & $0.93-1.23$ & 39.7 & 0.98 & $0.88-1.09$ & 31.7 & 0.99 & $0.88-1.11$ & 14.6 & 1.01 & $0.87-1.17$ & 8.7 & 1.00 & $0.83-1.21$ \\
\hline $36-49(708)$ & 14.6 & 0.97 & $0.80-1.17$ & 36.7 & 0.92 & $0.79-1.06$ & 34.0 & 1.02 & $0.87-1.19$ & 12.3 & 0.91 & $0.74-1.11$ & 8.2 & 0.95 & $0.74-1.22$ \\
\hline \multicolumn{16}{|l|}{ Maternal level of education } \\
\hline No education (1593) & 20.2 & 0.95 & $0.79-1.14$ & 38.0 & 0.92 & $0.79-1.06$ & 35.2 & 0.98 & $0.84-1.15$ & 17.6 & & Ref & 11.4 & 0.86 & $0.67-1.10$ \\
\hline Primary $(7560)$ & 16.5 & Ref & & 38.7 & Ref & & 34.9 & Ref & & 14.1 & 0.95 & $0.78-1.16$ & 8.9 & Ref & \\
\hline Secondary/Higher (3008) & 16.2 & 1.06 & $0.92-1.23$ & 41.4 & 1.02 & $0.91-1.14$ & 27.5 & $0.80^{\mathrm{c}}$ & $0.71-0.91$ & 13.0 & 1.05 & $0.90-1.23$ & 6.9 & 0.87 & $0.71-1.06$ \\
\hline \multicolumn{16}{|l|}{ Household wealth index } \\
\hline Lowest (3151) & 20.3 & Ref & & 39.1 & $\operatorname{Ref}$ & & 41.8 & Ref & & 17.6 & & Ref & 12.3 & Ref & \\
\hline Low (2678) & 16.7 & 1.05 & $0.89-1.24$ & 36.9 & 0.99 & $0.87-1.13$ & 35.3 & 0.96 & $0.84-1.10$ & 14.2 & 1.03 & $0.87-1.23$ & 9.0 & 1.04 & $0.84-1.28$ \\
\hline Middle (2362) & 16.1 & 1.04 & $0.87-1.25$ & 39.3 & 1.05 & $0.91-1.21$ & 31.5 & 1.07 & $0.92-1.24$ & 13.5 & 0.98 & $0.81-1.20$ & 7.3 & 0.96 & $0.75-1.23$ \\
\hline High (2066) & 16.3 & 1.09 & $0.89-1.33$ & 41.4 & 1.06 & $0.91-1.25$ & 29.3 & 0.97 & $0.81-1.14$ & 13.9 & 1.08 & $0.87-1.34$ & 7.7 & 1.14 & $0.87-1.48$ \\
\hline Highest (1922) & 11.7 & 0.78 & $0.59-1.04$ & 40.6 & 0.92 & $0.74-1.13$ & 18.8 & $0.64^{\mathrm{c}}$ & $0.51-0.81$ & 10.1 & 0.75 & $0.55-1.00$ & 4.5 & 0.68 & $0.46-1.02$ \\
\hline \multicolumn{16}{|l|}{ Household cooking location $¥$} \\
\hline Indoors (9154) & 16.4 & Ref & & 38.7 & Ref & & 33.3 & Ref & & 13.9 & & Ref & 8.4 & $\operatorname{Ref}$ & \\
\hline \multirow{2}{*}{\multicolumn{16}{|c|}{ Number of household members (3972) }} \\
\hline & & & & & & & & & & & & & & & \\
\hline $1-4(3589)$ & 16.4 & Ref & & 40.4 & Ref & & 30.0 & Ref & & 14.2 & & Ref & 7.5 & Ref & \\
\hline $5-8(6460)$ & 17.0 & 0.97 & $0.85-1.11$ & 39.3 & 1.00 & $0.90-1.10$ & 32.7 & 0.94 & $0.84-1.06$ & 14.6 & 0.98 & $0.85-1.13$ & 9.0 & 1.07 & $0.89-1.28$ \\
\hline $8-27(2130)$ & 16.0 & 0.89 & $0.74-1.06$ & 37.3 & $0.87^{\mathrm{a}}$ & $0.75-0.99$ & 36.8 & 0.90 & $0.78-1.05$ & 13.4 & 0.89 & $0.74-1.07$ & 9.2 & 1.01 & $0.80-1.28$ \\
\hline \multicolumn{16}{|l|}{ Survey season } \\
\hline Wet (4649) & 15.9 & Ref & & 36.7 & Ref & & 32.3 & Ref & & 13.6 & & Ref & 8.3 & Ref & \\
\hline Dry (7330) & 17.1 & 1.09 & $0.98-1.23$ & 41.0 & $1.18^{\mathrm{c}}$ & $1.08-1.28$ & 32.8 & 1.03 & $0.93-1.13$ & 14.7 & 1.09 & $0.97-1.23$ & 8.8 & 1.11 & $0.95-1.29$ \\
\hline
\end{tabular}

a $p<0.05 ;^{\mathrm{b}} p<0.01{ }^{\mathrm{c}} p<0.001,+$ Number of cases, with percentage of cases to non-cases with each category, OR = Adjusted odd ratio, CI $=95 \%$ confidence interval. Ref. $=$ reference category. $¥$ Variable has missing observations: Vitamin A supplementation in last 6 months $=84(0.7 \%)$, Mode of delivery $=49(0.4 \%)$. Not associated in the multivariate model $(p>0.05)=$ Birth order, Place of residence, Household smoking, Taking Iron pills, sprinkles or syrup. 
Table 3. Adjusted logistic regression analyses for urban and rural study sub-populations ( $\mathrm{N}=2426, \mathrm{~N}=10,840)$.

\begin{tabular}{|c|c|c|c|c|c|c|c|c|c|c|c|c|c|c|c|c|c|c|c|c|c|c|c|c|}
\hline \multirow{2}{*}{ Predictors $(N)$} & \multicolumn{6}{|c|}{ Shortness of Breath } & \multicolumn{6}{|c|}{ Cough } & \multicolumn{6}{|c|}{ Fever } & \multicolumn{6}{|c|}{$\begin{array}{ll}\text { Severe ARI } \\
\end{array}$} \\
\hline & \multicolumn{3}{|c|}{ Urban $(\mathbf{N}=\mathbf{2 2 1 9 )}$} & \multicolumn{3}{|c|}{$\begin{array}{c}\quad \text { Rural (N }=12,161) \\
\%+\quad O R\end{array}$} & \multicolumn{3}{|c|}{ Urban $(\mathrm{N}=2220)$} & \multicolumn{3}{|c|}{ Rural $(\mathrm{N}=12,163)$} & \multicolumn{3}{|c|}{$\operatorname{Urban}(\mathrm{N}=2220)$} & \multicolumn{3}{|c|}{ Rural (N = 9959) } & \multicolumn{3}{|c|}{$\begin{array}{c}\text { Urban }(\mathrm{N}=2197) \\
+\quad{ }_{O R}={ }_{C I}\end{array}$} & \multicolumn{3}{|c|}{$\%+\underset{O R}{\text { Rural }(\mathbf{N}=9770)}$} \\
\hline \multicolumn{25}{|c|}{ Household Cooking fuel } \\
\hline $\begin{array}{l}\text { Charcoal } \\
\text {. }\end{array}$ & 10.9 & Ref. & & 13.0 & Ref. & & 40.2 & Ref & & 40.0 & Ref. & & 18.4 & Ref. & & 29.0 & Ref. & & 4.6 & Ref. & & 6.8 & Ref. & \\
\hline Wood & 18.9 & $1.84^{\mathrm{b}}$ & $1.20-2.83$ & 17.7 & 1.22 & $0.99-1.50$ & 42.4 & 1.16 & $0.84-1.58$ & 39.1 & 1.22 & $0.99-1.50$ & 26.3 & 1.35 & $0.93-1.95$ & 36.0 & $1.23^{\mathrm{a}}$ & $1.03-1.47$ & 9.8 & 1.77 & $0.97-3.20$ & 9.4 & 1.33 & $0.99-1.80$ \\
\hline \multicolumn{6}{|l|}{$\begin{array}{l}\text { Wood } \\
\text { Child age }\end{array}$} & $0.96-1.33$ & 32.9 & $0.61^{\mathrm{c}}$ & $0.46-0.82$ & & 0.89 & $0.78-1.01$ & 18.2 & $0.66^{\mathrm{b}}$ & $0.43-0.87$ & 32.3 & $0.69^{\mathrm{c}}$ & $0.60-0.80$ & 5.9 & 0.73 & $0.41-1.28$ & 9.7 & 0.97 & $0.78-1.20$ \\
\hline $12-23$ & & Ref. & $0.01-1$. & 19.5 & Ref. & & 47.6 & Ref. & & 45.8 & Ref. & & 27.8 & & & 41.1 & Ref & & 8.0 & Ref. & & 11.4 & Ref & \\
\hline & & 0.87 & $0.59-1.30$ & 18.2 & 0.94 & $0.80-1.11$ & 42.4 & 0.90 & $0.68-1.20$ & 42.2 & 0.90 & $0.79-1.03$ & 21.6 & $0.69^{\mathrm{a}}$ & $0.49-0.96$ & 38.5 & 89 & $0.77-1.03$ & 6.7 & 88 & $0.51-1.51$ & 10.1 & 0.87 & $0.70-1.08$ \\
\hline $36-47$ & & 0. & & .5 & $0.60^{\mathrm{c}}$ & 2 & & 0.9 & 27 & 38.5 & $0.74^{\mathrm{c}}$ & 64-0.85 & & $0.71^{\mathrm{a}}$ & 1.00 & 34.2 & $0.73^{\mathrm{c}}$ & & 8.6 & 0.73 & & 7.6 & $0.64^{\mathrm{c}}$ & \\
\hline \multirow{2}{*}{\multicolumn{25}{|c|}{$\begin{array}{c}48-59 \\
\text { Child sex }\end{array}$}} \\
\hline & 12.7 & & & 15.9 & Ref. & & & Ref. & & 38.9 & & & 20.5 & & & & & & & & & & & \\
\hline \multirow{2}{*}{\multicolumn{25}{|c|}{$\begin{array}{l}\text { Mode of delivery } ¥ \\
\text { Mode }\end{array}$}} \\
\hline & & & & & & & & & & & & & & & & & & & & & & & & \\
\hline Caesarean & 9.2 & Ref. & & 16.9 & Ref. & & 40.2 & Ref. & & 36.9 & Ref. & & 8.7 & Ref. & & 26.1 & Ref. & & 4.3 & Ref. & & 5.3 & Ref. & \\
\hline Vaginal & 90.8 & 1.20 & $0.76-1.97$ & 12.4 & $1.46^{\mathrm{a}}$ & $1.07-2.03$ & 41.1 & 1.13 & $0.82-1.56$ & 39.5 & $1.33^{\mathrm{a}}$ & $1.06-1.68$ & 91.3 & 1.28 & $0.85-1.99$ & 35.6 & $1.42^{\mathrm{b}}$ & $1.10-1.87$ & 6.7 & 1.19 & $0.61-2.56$ & 9.3 & $1.64^{\mathrm{a}}$ & $1.04-2.74$ \\
\hline Vitamin A sup & ast $6 r$ & onths $¥$ & & 10 & & & & & & & & & & & & & & & & & & & & \\
\hline $\begin{array}{l}\text { No } \\
\text { Yes }\end{array}$ & 12.2 & Ref. & $38-1$ & $\begin{array}{l}14.1 \\
18.7\end{array}$ & $\begin{array}{l}\text { Ref. } \\
1.49^{\circ}\end{array}$ & $2-1$ & & $\begin{array}{l}\text { Ref. } \\
1.77^{\circ}\end{array}$ & $40-2$ & $\begin{array}{l}33.0 \\
44.3\end{array}$ & $\begin{array}{l}\text { Ref. } \\
1.60^{\circ}\end{array}$ & $1.52-1$ & $\begin{array}{l}16.3 \\
25.2\end{array}$ & $\begin{array}{l}\text { Ref. } \\
169 \mathrm{c}\end{array}$ & $130-20$ & $\begin{array}{l}31.2 \\
38.3\end{array}$ & $\begin{array}{l}\text { Ref. } \\
146 \mathrm{c}\end{array}$ & $131-16$ & 5.6 & Ref. & $086-200$ & $\begin{array}{l}6.9 \\
10.8\end{array}$ & $\begin{array}{l}\text { Ref. } \\
17^{\mathrm{c}}\end{array}$ & $.47-2.06$ \\
\hline Breastfe & & & 0 & & & $2-1$ & & & & & & $32-1$ & & & $30-2.20$ & & & $31-1.02$ & & & $80-2.00$ & & & \\
\hline Ever & 13.9 & Ref. & & 16.7 & Ref. & & 41.2 & Ref. & & 39.4 & Ref. & & 21.4 & Ref. & & 35.2 & Ref. & & 6.5 & Ref. & & 9.1 & Ref. & \\
\hline $\begin{array}{l}\text { Neve } \\
\text { Matern }\end{array}$ & 9.3 & 0.71 & $0.21-1.86$ & 13.7 & 0.84 & $0.49-1.36$ & 32.1 & 0.56 & $0.27-1.10$ & 32.0 & 0.76 & $0.53-1.10$ & 15.1 & 0.86 & $0.33-1.96$ & 32.5 & 0.93 & $0.62-1.37$ & 3.8 & 0.91 & $0.14-3.22$ & 6.8 & 0.67 & $0.28-1.36$ \\
\hline $\begin{array}{l}15-24 \\
15\end{array}$ & 15.6 & Ref. & & 17.0 & Ref. & & 43.4 & & & 39.9 & Ref. & & 24.3 & & & 35.2 & Ref. & & 8.1 & Ref. & & 11.1 & Ref. & \\
\hline & & 0.79 & & 17.1 & 1.14 & & 40 & & & 39.7 & 1.04 & & & & & & & & & & & 9.1 & & \\
\hline & 10.3 & $0.48^{\mathrm{a}}$ & $0.27-$ & 14.6 & 1.08 & $0.88-1$ & 37.6 & $0.66^{\mathrm{a}}$ & $0.45-0.95$ & 36.7 & 0.98 & $0.83-1$ & 21.9 & 0.87 & $0.55-1.36$ & 36.2 & 1.04 & $0.87-1.23$ & 5.5 & 0.51 & $0.23-1.06$ & 7.5 & 1.05 & $0.80-1.37$ \\
\hline Mate & & & $044-17$ & & & $078-115$ & & & $071-201$ & & & $077-105$ & & & $0.33-1.12$ & & & $0.86-1.19$ & & & & & & $64-1.08$ \\
\hline & & & $0.44-1.76$ & $\begin{array}{l}15.9 \\
61.5\end{array}$ & $\begin{array}{l}0.95 \\
\text { Ref. }\end{array}$ & $0.78-1.15$ & $\begin{array}{l}42 \\
41\end{array}$ & & 71-2.01 & $\begin{array}{l}38.0 \\
38.7\end{array}$ & $\begin{array}{l}0.90 \\
\text { Ref }\end{array}$ & $0.77-1.05$ & $\begin{array}{l}8.9 \\
48.1\end{array}$ & & 33-1.21 & $\begin{array}{l}14.8 \\
69.0\end{array}$ & & $86-1.19$ & & & & $\begin{array}{l}1.7 \\
67.0\end{array}$ & 84. & $.64-1.08$ \\
\hline & 41.2 & 0.76 & $0.55-1.06$ & 22.6 & 1.14 & $0.97-1.34$ & 40.3 & 0.93 & $0.74-1.18$ & 41.4 & 1.05 & $0.92-1.19$ & 43.0 & $0.69^{\mathrm{a}}$ & $0.52-0.92$ & 16.2 & $0.82^{b}$ & $0.71-0.94$ & 36.6 & 0.75 & $0.47-1.21$ & 15.3 & 0.90 & $0.71-1.12$ \\
\hline $\begin{array}{r}\text { House } \\
\text { Low }\end{array}$ & 24.4 & & & 20.3 & Ref & & 41.6 & Reff & & 39.1 & Ref. & & 35.7 & & & 42.3 & & & 15.4 & Ref. & & 12.1 & Ref. & \\
\hline & & & & & & & & & & & & & & & & & & & & & & & & \\
\hline & & & & & & & & & & & & & & & & & & & & & & & & \\
\hline & & & & & & & & & & & & & & & & & & & & & & & & \\
\hline High & 11.6 & & $0.56-2.10$ & 11.7 & $0.64^{a}$ & $0.45-0.89$ & 42.0 & & $0.72-1.99$ & 40.6 & $0.78^{a}$ & $0.61-1.00$ & 17.1 & 0.80 & $0.45-1.42$ & 22.3 & $0.58^{\mathrm{c}}$ & $0.43-0.76$ & & 0.89 & $0.37-2.17$ & 4.7 & $0.58^{\mathrm{a}}$ & $0.34-0.95$ \\
\hline Indoo & 14 & $R$ & & 16 & & & & & & 38 & & & 21 & & & 35.3 & & & 6 & & & 8.7 & & \\
\hline & 13.0 & 0.87 & $0.64-1.18$ & 17.2 & 1.06 & $0.91-1.24$ & 39.4 & 0.95 & $0.76-1.18$ & 41.1 & $1.20^{\mathrm{b}}$ & $1.06-1.35$ & 21.5 & 0.97 & $0.74-1.27$ & 34.2 & 1.11 & $0.97-1.26$ & 6.3 & 0.95 & $0.61-1.47$ & 10.6 & 1.15 & $0.94-1.41$ \\
\hline ber of ho & & & & & & & & & & & & & & & & & & & & & & & & \\
\hline $\begin{array}{l}1- \\
5-\end{array}$ & 14 & & & & & & & & & & & & & & & & & & & & & & & \\
\hline & 11.1 & 0.65 & $0.36-1$ & 16.0 & 0.92 & $0.76-1$ & 37.3 & 0.97 & $0.67-1.40$ & 36.7 & $0.85^{\mathrm{a}}$ & $0.73-0.98$ & 22.1 & 0.82 & $0.52-1.27$ & 38.7 & 0.92 & $0.78-1.08$ & 6.2 & 0.75 & $0.33-1.58$ & 9.5 & 1.03 & $0.80-1.32$ \\
\hline & & & & & & & & & & & & & & & & & & & & & & & & \\
\hline $\mathrm{W}$ & 12.1 & & & 15.9 & Ref. & & & & & 36.7 & a & & & Kej & & . & & & 4.7 & Kag. & & 9.2 & $\begin{array}{l}\text { Ref. } \\
\text { Ret }\end{array}$ & \\
\hline$D_{t}$ & 15.0 & 1.23 & $0.90-1.67$ & 17.1 & 1.07 & $0.95-1.22$ & 45.2 & $1.62^{\mathrm{c}}$ & 31-2.01 & 41.0 & $1.11^{\mathrm{a}}$ & $1.01-1.22$ & 24.5 & $1.51^{\mathrm{b}}$ & $1.15-1.99$ & 34.6 & 0.9 & $0.86-1.07$ & 7.8 & $1.74^{\mathrm{a}}$ & $1.10-2.82$ & 9.0 & 1.04 & $0.89-1.23$ \\
\hline
\end{tabular}

a $p<0.05 ;{ }^{\mathrm{b}} p<0.01{ }^{\mathrm{c}} p<0.001 ; \mathrm{N}=$ Number of observations within category. + Percentage of cases to non-cases within each category, OR $=$ Adjusted odd ratio, $\mathrm{CI}=95 \%$ confidence interval. Ref. $=$ reference category. $¥$ Variable has missing observations: Taking Iron supplements $=58(0.6 \%)$, Cooking location $=16(0.1 \%)$, Mode of Delivery $=41(0.4 \%)$. Not associated in the multivariate model $(p>0.05)=$ Birth order, household smoking, cooking location. 


\section{Discussion}

Globally, almost half of the world's population cook with solid biomass fuels; associated with pollutant concentrations which typically exceed WHO Indoor Air Quality Guidelines [27]. Although traditional combustion of charcoal cooking fuel is recognized to emit lower air pollutant concentrations compared to firewood, there is a paucity of evidence regarding respiratory health benefits of charcoal biomass fuel alternatives and no previous evidence from Uganda, where over $96 \%$ of the population use biomass fuels as the primary domestic energy source [15]; compounded by health and social inequality as a result of barriers to cleaner fuel access.

This observational, nationally-representative population based study, with a large sample size ( $n=15,522)$ and high response rate $(98 \%)$ has identified that wood fuel cooking is associated with increased individual risk of respiratory symptoms and ARI risk among children aged under 5 years living in biomass fuel households in urban and rural settings in Uganda. Children are recognized to be at increased vulnerability to HAP exposure and ARI is a leading causes of death among those aged under 5 years worldwide, contributing to an estimated 1.9-2.2 million deaths worldwide each year [28]; therefore prevention could have wide reaching health and fiscal benefits.

Our observations regarding the increased respiratory risks associated with wood compared to charcoal biomass fuel are broadly consistent with current evidence from low income contexts [29,30]. Sana et al. [31] showed that women living within an urban population in Burkina Faso had increased risk of cough among those using wood, compared to charcoal cooking. Taylor and Nakai [32] also observed ARI prevalence to be higher for children in homes with wood stoves compared to charcoal stoves in Sierra Leone, and overall ARI prevalence was higher than reported previously. However, this study was undertaken in the rainy season in Sierra Leone, which is recognized to influence the occurrence of ARI and ARI symptoms [32]. Our study showed a significant association with season (two seasons observed over 6 months), with the dry season (June to August) increasing the risk of cough in both urban and rural settings.

Our study has a number of limitations; the DHS survey includes questions only about fuel type and we did not have available information regarding other fuels used to light the stove or mixed fuel use. Charcoal is recognized to be difficult to ignite, therefore a starting fuel is typically required, which may comprise wood, straw or crop residues in rural areas, with plastics or kerosene more likely to be used in urban areas, therefore influencing exposure to specific air pollutants include volatile organic compounds (VOCs) [31]. However, wood combustion is consistently associated with high concentrations of particulate matter [33-37], which is likely to be the dominant pollutant in biomass fuel domestic settings and which has a causal relationship with ARI risk in other sub-Saharan settings [38-40]. Furthermore, detailed information was not available for wood and charcoal fuel types (such as tree species, seasoning), family cooking practices or fuel usage patterns and mixing, which limits comparison of findings between study populations, or those which rely upon fuel type as a proxy for air pollutant exposure.

ARI risk is also recognised to be caused by cumulative long-term pollutant exposure [32], which cannot be determined in this cross-sectional study. However, evidence suggests that fuel choices and cooking remain relatively consistent among biomass fuel households in low-income contexts [4]. Therefore, similar levels of chronic exposure would be expected for those using a specific biomass fuel type. Another contributory factor is household smoking, which has a low prevalence in Uganda $(16.4 \%)$ [41], and within this study $13.3 \%$ of children resided in a household with a smoking household member; reflecting the important contribution of HAP to respiratory disease risk in this context. Although outdoor cooking and ventilation has been shown to decrease HAP levels [42], it may not be below the WHO level; which may explain why there is an increased risk of cough with outdoor cooking. Our use of DHS data at the household and individual maternal and child levels enabled adjustment for a wide range of socio-demographic, household and contextual factors known to be associated with the risk of respiratory symptoms, including socio-economic status (wealth index), season, region, household size, maternal level of education, breastfeeding status, mode of delivery and 
vitamin A supplementation and birth order. However, these factors may be subject to recall bias and detailed information was not available for co-inhabitation with livestock, cooking activity patterns, or other sources of domestic or ambient pollution; however, our findings reflect the widespread use of fuel type as a proxy indicator and which is meaningful and easily communicated to families in this context.

Biomass is the primary domestic fuel in sub-Saharan Africa (76.7\%) [43], which remains the world region with the highest burden arising from household air pollution. In the context of limited availability of cleaner fuels mitigation measures have potential large scale public health benefit for primary prevention of respiratory symptoms and disease in this setting. The energy ladder presents the transition phase from solid biomass and readily collected fuels (such as wood, dung, straw) through processed natural fuels (e.g., charcoal) to supplied "cleaner" fuels (e.g., electricity, LPG); associated with shift to an industrialized energy economy [44]. In experimental setting, charcoal, although still presenting some health risks [31] was associated with lower levels of $\mathrm{CO}, \mathrm{CO}_{2}$ and $\mathrm{PM}_{2.5}$ compared to wood [45]; however these vary according to moisture content, wood type and circumstances of combustion (among other factors). In addition, similar to charcoal are briquettes, another potential transition towards cleaner fuel, are associated with lowered HAP levels, but still exceed the WHO guidelines levels [46]; and require further research into the respiratory benefit.

DHS data is based upon self-reported respiratory symptoms, which can be subject to recall bias, and our primary outcome measure does not represent an objective clinical ARI diagnosis. Therefore, future research is need to determine the air quality and health benefits arising from differences in fuel type, utilising objective air quality assessment and including information on chronic health conditions, cooking activity behaviours, household layout stove design and ventilation. In addition, future DHS surveys and research which includes detailed individual level information on pre-existing health conditions would enable assessment of the role of pre-existing vulnerability, such as the recognised associations between TB [28], HIV [47], ARI severity [47] and poverty [48,49]. Furthermore, epidemiological studies evaluating the impacts of solid biomass fuels should capture the wider environmental impacts, physical hazards and opportunity costs associated with wood collection and charcoal production [50]; along with the occurrence of childhood morbidities affecting further life chances.

Our novel findings suggest potential benefits arising from transition from wood to charcoal fuels in low-income contexts where financial and logistical barriers limit access to cleaner domestic fuels; particularly among those families living in urban areas. A harm mitigation policy approach could be adopted to incentivize transition away from wood to cleaner biomass fuels (such as processed high quality charcoal and briquettes) whilst supporting traditional cooking activity practices and therefore enabling sustained uptake. Major national initiatives to facilitate cleaner fuel use have been frequently unsuccessful due to a lack of socio-cultural considerations, health awareness, education, affordability (fuels, stoves) and supply chain issues [51,52]. In order for improvement in respiratory health to occur, policy makers need to focus on clean fuel switching taking into consideration other confounding factors and vulnerabilities (wealth, breastfeeding, mothers education) found within this study and others [31,53-55], which can be modified through policy.

\section{Conclusions}

Replacing wood with charcoal cooking fuel may reduce the incidence of ARI respiratory symptoms shortness of breath, fever, cough, ARI and severe ARI in children aged under 5 years in urban Uganda. Although charcoal is typically more expensive than wood, public health policies applying a harm mitigation approach in contexts which lack cleaner fuel access, may reduce the HAP associated morbidity and mortality burden among young children in this setting. 
Author Contributions: Conceptualization, T.B. and S.E.B.; data curation, K.E.W.; methodology, T.B., K.E.W., G.N.T. and S.E.B.; formal analysis, K.E.W.; writing-original draft preparation, T.B., K.E.W. and S.E.B.; writing-review and editing, A.S., S.T.M., B.K., T.K., A.W., G.N.T., W.R.A. and F.D.P.; visualization, K.E.W.; supervision, S.E.B., G.N.T. and F.D.P. All authors have read and agreed to the published version of the manuscript.

Funding: The work is funded by the UK Department for International Development (DFID) via the East Africa Research Fund (EARF) grant 'A Systems Approach to Air Pollution (ASAP) East Africa.

Acknowledgments: We are grateful for being granted permission and gaining access to the Demographic Health survey data. In addition, we would like to acknowledge the University of Birmingham for covering the article processing charge and provision of a Global Challenges Scholarship to K.W.

Conflicts of Interest: The authors declare no conflict of interest.

\section{References}

1. Chafe, Z.; Brauer, M.; Klimont, Z.; Van Dingenen, R.; Mehta, S.; Rao, S.; Riahi, K.; Dentener, F.; Smith, K. Household Cooking with Solid Fuels Contributes to Ambient PM2. Environ. Health Perspect. 2014, 122, 1314-1320. [CrossRef]

2. World Health Organization (WHO). Household Air Pollution and Health. Available online: https://www. who.int/news-room/fact-sheets/detail/household-air-pollution-and-health (accessed on 24 January 2020).

3. Ezzati, M.; Kammen, D.M. The Health Impacts of Exposure to Indoor Air Pollution from Solid Fuels in Developing Countries: Knowledge, Gaps, and Data Needs. Environ. Health Perspect. 2002, 110, 1057-1068. [CrossRef] [PubMed]

4. Kurmi, O.P.; Bong, K.; Lam, H.; Ayres, J.G. Indoor Air Pollution and the Lung in Low- and Medium-Income Countries. Eur. Respir. 2012, 40, 239-254. [CrossRef]

5. Oluwole, O.; Arinola, G.O.; Huo, D.; Olopade, C.O. Household Biomass Fuel Use, Asthma Symptoms Severity, and Asthma Underdiagnosis in Rural Schoolchildren in Nigeria: A Cross-Sectional Observational Study. BMC Pulm. Med. 2017, 17, 3. [CrossRef]

6. Kurmi, O.P.; Semple, S.; Devereux, G.S.; Gaihre, S.; Bong, K.; Lam, H.; Sadhra, S.; Steiner, M.F.; Simkhada, P.; Smith, W.C.; et al. The Effect of Exposure to Biomass Smoke on Respiratory Symptoms in Adult Rural and Urban Nepalese Populations. Environ. Health 2014, 13, 92. [CrossRef]

7. Bruce, N.; Dherani, M.; Liu, R.; Hosgood, H.D.; Sapkota, A.; Smith, K.R.; Straif, K.; Lan, Q.; Pope, D. Does Household Use of Biomass Fuel Cause Lung Cancer? A Systematic Review and Evaluation of the Evidence for the GBD 2010 Study. Thorax 2015, 70, 433-441. [CrossRef]

8. Sood, A.; Assad, N.A.; Barnes, P.J.; Churg, A.; Gordon, S.B.; Harrod, K.S.; Irshad, H.; Kurmi, O.P.; Ii, W.J.M.; Meek, P.; et al. ERS/ATS Workshop Report on Respiratory Health Effects of Household Air Pollution. Eur. Respir. J. 2017, 51, 1700698. [CrossRef]

9. Das, I.; Jagger, P.; Yeatts, K. Biomass Cooking Fuels and Health Outcomes for Women in Malawi. Ecohealth 2017, 14, 7-19. [CrossRef]

10. Khalequzzaman, M.; Kamijima, M.; Sakai, K.; Ebara, T.; Hoque, B.A.; Nakajima, T. Indoor Air Pollution and Health of Children in Biomass Fuel-Using Households of Bangladesh: Comparison between Urban and Rural Areas. Environ. Health Prev. Med. 2011, 16, 375-383. [CrossRef]

11. Pollard, S.L.; Williams, A.L.; Breysse, P.N.; Baron, P.A.; Grajeda, L.M.; Gilman, R.H.; Jaime Miranda, J.; Checkley, W. A Cross-Sectional Study of Determinants of Indoor Environmental Exposures in Households with and without Chronic Exposure to Biomass Fuel Smoke. Environ. Health 2014, 13, 21. [CrossRef]

12. The World Bank Group. Uganda | Data. Available online: https://data.worldbank.org/country/uganda (accessed on 23 January 2020).

13. The World Bank Group. Urban Population-Uganda | Data. Available online: https://data.worldbank.org/ indicator/SP.URB.TOTL?locations=UG (accessed on 23 January 2020).

14. Gordon, S.B.; Bruce, N.G.; Grigg, J.; Hibberd, P.L.; Kurmi, O.P.; Lam, K.H.; Mortimer, K.; Asante, K.P.; Balakrishnan, K.; Balmes, J.; et al. Respiratory Risks from Household Air Pollution in Low and Middle Income Countries. Lancet Respir. Med. 2014, 2, 823-860. [CrossRef]

15. NEMA. State of the Environment Report for Uganda 2014; National Environment Management Authority (NEMA): Kampala, Uganda, 2016.

16. Global Burden of Disease. GBD Compare | IHME Viz Hub. Available online: https://vizhub.healthdata.org/ gbd-compare/ (accessed on 27 January 2020). 
17. Van Gemert, F.; Chavannes, N.; Kirenga, B.; Jones, R.; Williams, S.; Tsiligianni, I.; Vonk, J.; Kocks, J.; De Jong, C.; Van Der Molen, T. Socio-Economic Factors, Gender and Smoking as Determinants of COPD in a Low-Income Country of Sub-Saharan Africa: FRESH AIR Uganda. Prim. Care Respir. Med. 2016, 26, 1-6. [CrossRef] [PubMed]

18. Van Gemert, F.; Kirenga, B.; Chavannes, N.; Kamya, M.; Luzige, S.; Musinguzi, P.; Turyagaruka, J.; Jones, R. Prevalence of chronic obstructive pulmonary disease and associated risk factors in Uganda (fresh air Uganda): A prospective cross-sectional observational study. Lancet Glob. Health 2015, 3, e44-e51. [CrossRef]

19. Simoes, E.A.F.; Cherian, T.; Chow, J.; Shahid-Salles, S.A.; Laxminarayan, R.; John, T.J. Acute Respiratory Infections in Children. In Disease Control Priorities in Developing Countries, 2nd ed.; Jamison, D.T., Breman, J.G., Measham, A.R., Alleyne, G., Claeson, M., Evans, D.B., Jha, P., Mills, A., Musgrove, P., Eds.; The International Bank for Reconstruction and Development/The World Bank: Washington, DC, USA, 2006; Chapter 25.

20. The DHS Program. Uganda: Standard DHS. 2016. Available online: https://dhsprogram.com/what-we-do/ survey/survey-display-504.cfm (accessed on 24 January 2020).

21. Uganda Bureau of Statistics (UBOS). Uganda Demographic and Health Survey 2016; ICF: Kampala, Uganda, 2018.

22. Croft, T.N.; Marshall, A.M.J.; Allen, C.K.; Arnold, F.; Assaf, S.; Balian, S. Guide to DHS Statistics; ICF: Rockville, MD, USA, 2018.

23. World Health Organization (WHO). WHO Global Technical Consultation: Global Standards and Tools for Influenza Surveillance; World Health Organization (WHO): Geneva, Switzerland, 2011.

24. Km, R.; Gupta, V.; Ahmad, S.; Ranhotra, S.; Issrani, R.; Prabhu, N. Assessment of Anemia as a Risk Factor for Acute Lower Respiratory Tract Infections in Children: A Case-Control Study. Int. J. Clin. Pediatr. 2015, 4, 149-153. [CrossRef]

25. Central Intelligence Agency (CIA). The World Factbook. Available online: https://www.cia.gov/library/ publications/the-world-factbook/ (accessed on 31 January 2020).

26. RStudio Team. RStudio: Integrated Development for R; RStudio, Inc.: Boston, MA, USA, 2015.

27. World Health Organization (WHO). WHO Guidelines for Indoor Air Quality: Selected Pollutants; World Health Organization (WHO): Copenhagen, Denmark, 2010.

28. Madhi, S.A.; Klugman, K.P. Acute Respiratory Infections. In Disease and Mortality in Sub-Saharan Africa; Makgoba, M., Hofman, K.J., Feacham, R.G.A., Bainga, F., Khama, O.R., Bos, E.R., Jamison, D.T., Eds.; The International Bank for Reconstruction and Development/The World Bank: Washington, DC, USA, 2006; p. 149.

29. Fullerton, D.G.; Semple, S. Air Pollution and Health: Indoor Air Pollution in the Developing World Is the Real Key to Reducing the Burden of Ill Health. Thorax 2008, 63, 288.

30. Mishra, V. Indoor Air Pollution from Biomass Combustion and Acute Respiratory Illness in Preschool Age Children in Zimbabwe. Int. J. Epidemiol. 2003, 32, 847-853. [CrossRef]

31. Sana, A.; Meda, N.; Badoum, G.; Kafando, B.; Bouland, C. Primary Cooking Fuel Choice and Respiratory Health Outcomes among Women in Charge of Household Cooking in Ouagadougou, Burkina Faso: Cross-Sectional Study. Int. J. Environ. Res. Public Health 2019, 16, 1040. [CrossRef]

32. Taylor, E.T.; Nakai, S. Prevalence of Acute Respiratory Infections in Women and Children in Western Sierra Leone Due to Smoke from Wood and Charcoal Stoves. Int. J. Environ. Res. Public Health 2012, 9, 2252-2265. [CrossRef]

33. Bartington, S.E.; Bakolis, I.; Devakumar, D.; Kurmi, O.P.; Gulliver, J.; Chaube, G.; Manandhar, D.S.; Saville, N.M.; Costello, A.; Osrin, D.; et al. Patterns of Domestic Exposure to Carbon Monoxide and Particulate Matter in Households Using Biomass Fuel in Janakpur, Nepal. Environ. Pollut. 2017, 220, $38-45$. [CrossRef]

34. Tumwesige, V.; Okello, G.; Semple, S.; Smith, J. Impact of Partial Fuel Switch on Household Air Pollutants in Sub-Sahara Africa. Environ. Pollut. 2017, 231, 1021-1029. [CrossRef]

35. Ellegdard, A. Cooking Fuel Smoke and Respiratory Symptoms among Women in Low-Income Areas in Maputo. Environ. Health Perspect. 1996, 104, 980-985.

36. Titcombe, M.E.; Simcik, M.; Titcombe, M.E.; Simcik, M. Personal and Indoor Exposure to PM 2.5 and Polycyclic Aromatic Hydrocarbons in the Southern Highlands of Tanzania: A Pilot-Scale Study. Environ. Monit. Assess. 2011, 180, 461-476. [CrossRef] [PubMed]

37. Bede-Ojimadu, O.; Orisakwe, O.E. Exposure to Wood Smoke and Associated Health Effects in Sub-Saharan Africa: A Systematic Review. Ann. Glob. Health 2020, 86, 32. [CrossRef] 
38. Ezzati, M.; Kammen, D. Indoor Air Pollution from Biomass Combustion and Acute Respiratory Infections in Kenya: An Exposure-Response Study. Lancet 2001, 358, 619-624. [CrossRef]

39. Admasie, A.; Kumie, A.; Worku, A. Children under Five from Houses of Unclean Fuel Sources and Poorly Ventilated Houses Have Higher Odds of Suffering from Acute Respiratory Infection in Wolaita-Sodo, Southern Ethiopia: A Case-Control Study. J. Environ. Public Health 2018, 2018, 9320603. [CrossRef] [PubMed]

40. Sanbata, H.; Asfaw, A.; Kumie, A. Association of Biomass Fuel Use with Acute Respiratory Infections among Under-Five Children in a Slum Urban of Addis Ababa, Ethiopia. BMC Public Health 2014, 14, 1122. [CrossRef]

41. World Health Organization (WHO). Prevalence of Tobacco Smoking. Available online: http://gamapserver. who.int/gho/interactive_charts/tobacco/use/atlas.html (accessed on 27 January 2020).

42. Langbein, J. Firewood, Smoke and Respiratory Diseases in Developing Countries-The Neglected Role of Outdoor Cooking. PLoS ONE 2017, 12, e0178631. [CrossRef]

43. Owili, P.O.; Muga, M.A.; Pan, W.-C.; Kuo, H.-W. Cooking Fuel and Risk of Under-Five Mortality in 23 Sub-Saharan African Countries: A Population-Based Study). Int. J. Environ. Health Res. 2017, 27, 191-204. [CrossRef]

44. Smith, K.R.; Apte, M.G.; Yuqing, M.; Wongsekiarttirat, W.; Kulkarni, A. Air Pollution and the Energy Ladder in Asian Cities. Energy 1994, 19, 587-600. [CrossRef]

45. Obeng, G.Y.; Mensah, E.; Ashiagbor, G.; Boahen, O.; Sweeney, D.J. Watching the Smoke Rise up: Thermal Efficiency, Pollutant Emissions and Global Warming Impact of Three Biomass Cookstoves in Ghana. Energies 2017, 10, 641. [CrossRef]

46. Okello, G.; Mugisha, F.; Namaganda, I.; Avis, W. Understanding Household Fuel Transitions: The Case of Namuwongo (Kampala); University of Birmingham: Birmingham, UK, 2019.

47. Tazinya, A.A.; Halle-Ekane, G.E.; Mbuagbaw, L.T.; Abanda, M.; Atashili, J.; Obama, M.T. Risk Factors for Acute Respiratory Infections in Children under Five Years Attending the Bamenda Regional Hospital in Cameroon. BMC Pulm. Med. 2018, 18, 7. [CrossRef] [PubMed]

48. Harerimana, J.-M.; Nyirazinyoye, L.; Thomson, D.R.; Ntaganira, J. Social, Economic and Environmental Risk Factors for Acute Lower Respiratory Infections among Children under Five Years of Age in Rwanda. Arch. Public Health 2016, 74, 19. [CrossRef] [PubMed]

49. Yaya, S.; Bishwajit, G. Burden of Acute Respiratory Infections among Under-Five Children in Relation to Household Wealth and Socioeconomic Status in Bangladesh. Trop. Med. Infect. Dis. 2019, 4, 36. [CrossRef] [PubMed]

50. Jagger, P.; Shively, G.; Edu, S. Taxes and Bribes in Uganda. J. Dev. Stud. 2015, 51, 66-79. [CrossRef]

51. Puzzolo, E.; Stanistreet, D.; Pope, D.; Bruce, N.; Rehfuess, E. Factors Influencing the Large-Scale Uptake by Households of Cleaner and More Efficient Household Energy Technologies; EPPI-Centre, Social Science Research Unit, Institute of Education, University of London: London, UK, 2013.

52. Pillarisetti, A.; Jamison, D.T.; Smith, K.R. Household Energy Interventions and Health and Finances in Haryana, India: An Extended Cost-Effectiveness Analysis. In Disease Control; Priorities; Injury Prevention and Environmental Health, 3rd ed.; The World Bank: Washington, DC, USA, 2017; Volume 7, pp. 223-237.

53. Buchner, H.; Rehfuess, E.A. Cooking and Season as Risk Factors for Acute Lower Respiratory Infections in African Children: A Cross-Sectional Multi-Country Analysis. PLoS ONE 2015, 10, e0128933. [CrossRef]

54. Naz, S.; Page, A.; Agho, E.K. Household Air Pollution from Use of Cooking Fuel and Under-Five Mortality: The Role of Breastfeeding Status and Kitchen Location in Pakistan. PLoS ONE 2017, 12, e0173256. [CrossRef]

55. Avis, W.; Mariga, S.; Singh, A. Air Pollution Exposure in Low Income Households in Kampala; ASAP East Africa Vulnerability Scoping Study; No. 6; Birmingham; University of Birmingham: Birmingham, UK, 2018.

(C) 2020 by the authors. Licensee MDPI, Basel, Switzerland. This article is an open access article distributed under the terms and conditions of the Creative Commons Attribution (CC BY) license (http://creativecommons.org/licenses/by/4.0/). 